PROCEEDINGS OF THE

AMERICAN MATHEMATICAL SOCIETY

Volume 129, Number 7, Pages 1877-1883

S 0002-9939(01)06152-4

Article electronically published on February 22, 2001

\title{
COMPUTING MORDELL-WEIL RANKS OF CYCLIC COVERS OF ELLIPTIC SURFACES
}

\author{
LISA A. FASTENBERG
}

(Communicated by David E. Rohrlich)

\begin{abstract}
We give explicit formulas for computing the Mordell-Weil ranks of the elliptic surfaces $E_{r}: Y^{2}=X^{3}+a\left(t^{r}\right) X+b\left(t^{r}\right)$ subject to some restrictions on the surface $E_{1}$.
\end{abstract}

Let $\pi: E \rightarrow \mathbf{P}^{1}$ be a smooth complex relatively minimal elliptic surface with section. For each positive integer $r$, define $\pi_{r}: E_{r} \rightarrow \mathbf{P}^{1}$ to be the relatively minimal compactification of the Neron model of the generic fiber of $E \times \mathbf{P}^{1} \mathbf{P}^{1}$ of the pullback of $E$ by the morphism of $\mathbf{P}^{1}$ defined by $t \rightarrow t^{r}$. The main result of [F1, [F2] is that under certain conditions on $E$, the rank of the Mordell-Weil group of sections of $E_{r}$ is bounded independently of $r$. The purpose of this paper is to use this result to compute or give explicit upper bounds on the rank of $E_{r}\left(\mathbf{P}^{1}\right)$ for several examples.

We begin by showing that the following theorem is a consequence of the proof given in [F1], [F2]:

Theorem 1. Let $\pi: E \rightarrow \mathbf{P}^{1}$ be a nonisotrivial elliptic surface. For each $t \in \mathbf{P}^{1}$ let $E_{1}^{t}$ be the fiber of $E$ over $t$, let $f_{t}$ be the conductor of $E_{1}^{t}$, and let $e_{t}$ be the Euler characteristic of $E_{1}^{t}$. For $t=0$ and $\infty$, if $E_{1}^{t}$ is of type $I_{n}$ or $I_{n}^{*}$, let $n_{t}=n$. Otherwise, let $n_{t}=0$. Define

$$
\gamma=\sum_{t \neq 0, \infty}\left(f_{t}-e_{t} / 6\right)-\frac{n_{0}+n_{\infty}}{6}
$$

and assume that $\gamma<1$. For each positive integer $r$ let $\phi_{r}: \mathbf{P}^{1} \rightarrow \mathbf{P}^{1}$ be the morphism defined by $\phi_{r}(t)=t^{r}$, and let $E_{r}$ be the pullback of $E$ via $\phi_{r}$. Let $r=p_{1}^{m_{1}} \ldots p_{n}^{m_{n}}$. Then

$$
\operatorname{rank} E_{r}\left(\mathbf{P}^{1}\right) \leq \sum_{\substack{0 \leq l_{i} \leq m_{i} \\ p_{i}^{l_{i}}<2 /(1-\gamma)}} \phi\left(p_{1}^{l_{1}} \ldots p_{n}^{l_{n}}\right) .
$$

It is not known whether the rank of an elliptic surface over $\mathbf{P}^{1}$ is bounded. The largest known rank for an elliptic surface over $\mathbf{P}^{1}$ is 56 (see Example 1, and Sh2], St] $)$. We will see that we can often use a variant on the proof of Theorem 1 to get an explicit formula for the rank of the surfaces $E_{r}$, so that the technique outlined below can be used to look for elliptic surfaces with large rank. We have the following corollary to Theorem 1 .

Received by the editors April 8, 1999.

1991 Mathematics Subject Classification. Primary 14J27, 11G05.

(C)2001 American Mathematical Society 
Corollary. Let $E$ be an elliptic surface satisfying the conditions of Theorem 1. Then, for all $r, \operatorname{rank}\left(E_{r}\right) \leq 27720$.

Proof. If $\gamma<1$, then the largest possible value for $\gamma$ is $\gamma=5 / 6$, since $f_{t}, e_{t}$ and $n_{t}$ are all integers. If $\gamma=5 / 6$, then $2 /(1-\gamma)=12$ and we have that

$$
\operatorname{rank}\left(E_{r}\right) \leq \sum \phi(d)=27720
$$

where the sum is over all $d$, with $d=2^{l_{1}} 3^{l_{2}} 5^{l_{3}} 7^{l_{4}} 11^{l_{5}}$, and $l_{1} \in\{0,1,2,3\}, l_{2} \in$ $\{0,1,2\}$ and $l_{i} \in\{0,1\}$ for $i=3,4,5$.

The proof of Theorem 1 is contained in the next section. In Sections 2, 3 and 4 we show how to use the proof of Theorem 1 to improve on the bounds given there, and in some cases to explicitly compute the Mordell-Weil group of the elliptic surfaces $E_{r} / \mathbf{P}^{1}$.

\section{Proof of Theorem 1}

In this section we review the proof of Theorem 2 in [F1, state some minor corrections to the proof given there, and show how to extend it to a proof of Theorem 1. For additional details, see [F1. Let $E_{1}$ be an elliptic surface satisfying the conditions of Theorem 1, and let $f_{r, t}, e_{r, t}$ be the conductor and Euler characteristic of the fiber of $E_{r}$ over $t \in \mathbf{P}^{1}$ respectively. Note that $f_{r, t}$ is 0 if $E_{r}^{t}$ is nonsingular, 1 if $E_{r}^{t}$ is of multiplicative type, and 2 if $E_{r}^{t}$ is of additive type. For a list of the Euler characteristics of each of the possible Kodaira types, see Table (IV.3.1) of [Mi]. Let $k=m+2 a$ where $m, a$ are the number of singular fibers of $E_{1}$ over $\mathbf{P}^{1} \backslash\{0, \infty\}$ that have multiplicative or additive type respectively.

Recall that for any elliptic surface $\pi: E \rightarrow \mathbf{P}^{1}$, we have that [CZ]

$$
\operatorname{rank} E=\operatorname{dim} H^{1}\left(\mathbf{P}^{1}, R^{1} \pi_{*} \mathbf{Q}\right) \cap H^{1,1}(E, \mathbf{C}) .
$$

For $i+j=2$, define $H_{r}^{i, j}=H^{1}\left(\mathbf{P}^{1}, R^{1} \pi_{r *} \mathbf{C}\right) \cap H^{i, j}\left(E_{r}, \mathbf{C}\right)$. Then

$$
\begin{aligned}
\operatorname{rank} E_{1} & =\operatorname{dim} H^{1}\left(\mathbf{P}^{1}, R^{1} \pi_{1 *} \mathbf{Q}\right) \cap H^{1,1}\left(E_{1}, \mathbf{C}\right) \\
& \leq \operatorname{dim} H_{1}^{1,1}=-2+\sum_{t \in \mathbf{P}^{1}}\left(f_{1, t}-e_{1, t} / 6\right) .
\end{aligned}
$$

We can bound the rank of $E_{r}$ as follows:

$$
\begin{aligned}
\operatorname{rank} E_{r} & \leq \operatorname{dim}\left(H_{r}^{1,1}\right)=-2+\sum_{t \in \mathbf{P}^{1}}\left(f_{r, t}-e_{r, t} / 6\right) \\
& =-2+\sum_{t \in \mathbf{P}^{1} \backslash\{0, \infty\}}\left(f_{r, t}-e_{r, t} / 6\right)+\left(f_{r, 0}-e_{r, 0} / 6\right)+\left(f_{r, \infty}-e_{r, \infty} / 6\right) \\
& \leq 2+\sum_{t \in \mathbf{P}^{1} \backslash\{0, \infty\}}\left(r f_{1, t}-r e_{1, t} / 6\right)-r n_{0} / 6-r n_{\infty} / 6=2+r \gamma .
\end{aligned}
$$

The second inequality follows from the fact that for $t=0, \infty$ we have $f_{r, t} \leq 2$ and $e_{r, t} \geq r n_{t}$.

Now let $\sigma_{r}$ be the automorphism of $E_{r}$ which is lifted from the automorphism of $\mathbf{P}^{1}$ defined by $T \rightarrow \zeta_{r} T$, where $\zeta_{r}=e^{2 \pi i / r}$. Then $\sigma_{r}$ acts on the cohomology group $H^{1}\left(\mathbf{P}^{1}, R^{1} \pi_{r *} \mathbf{C}\right)$, preserving both the Hodge decomposition and rational cohomology. Let $N_{r}$ be the pushforward of the normal bundle to a section of $\pi_{r}$, and $N_{r, t}$ its stalk at $t$. For $t=0, \infty$, we have that $\operatorname{tr}\left(\sigma_{r}, N_{r, t}\right)=\zeta_{r}^{a_{t}}$ for some integer 
$a_{t}$, since $\sigma$ has order $r$ and $N_{r, t}$ is one dimensional. In [F1] we show that

$$
H^{1}\left(\mathbf{P}^{1}, R^{1} \pi_{r *} \mathbf{C}\right) \oplus H^{1}\left(E_{r}^{(0)}, \mathbf{C}\right) \oplus H^{1}\left(E_{r}^{(\infty)}, \mathbf{C}\right)=\mathbf{C}[\mathbf{Z} / r \mathbf{Z}]^{\oplus k},
$$

where $\mathbf{C}[\mathbf{Z} / r \mathbf{Z}]$, is the regular representation of $\mathbf{Z} / r \mathbf{Z}$. Furthermore, we show that, for some integer $l$,

$$
H_{r}^{0,2}=\mathbf{C}[\mathbf{Z} / r \mathbf{Z}]^{\oplus l} \oplus_{j=a_{\infty}+1}^{a_{0}-1} V_{j}, \quad H_{r}^{2,0}=\mathbf{C}[\mathbf{Z} / r \mathbf{Z}]^{\oplus l} \oplus_{j=-a_{o}+1}^{-a_{\infty}-1} V_{j},
$$

where $V_{j}$ is the 1-dimensional representation with eigenvalue $\zeta_{r}^{j}$. By assumption, $\gamma<1$, so that $\operatorname{dim}\left(H_{r}^{1,1}\right)<r$. This means that up to a vector space of dimension at most 4 the eigenvalues of $\sigma$ on $H_{r}^{1,1}$ are

$$
\zeta_{r}^{a}, \zeta_{r}^{a+1}, \ldots, \zeta_{r}^{b}, \zeta_{r}^{c}, \zeta_{r}^{c+1}, \ldots, \zeta_{r}^{d}
$$

where $a, b, c, d \in\left\{a_{0},-a_{0}, a_{\infty},-a_{\infty}\right\}$, each with one dimensional eigenspace, and no $\zeta_{r}^{j}$ appearing more than once. (Notice that the list of eigenvalues given in F1] may not always be correct, depending on $a_{0}, a_{\infty}$, but the final result is unchanged.)

If $d \mid r$, an eigenspace corresponding to a primitive $d$ th root of unity, is in $H^{1}\left(\mathbf{P}^{1}, R^{1} \pi_{1 *} \mathbf{Q}\right) \cap H_{r}^{1,1}$ only if all eigenspaces corresponding to primitive $d$ th roots of unity are also in $H^{1}\left(\mathbf{P}^{1}, R^{1} \pi_{1 *} \mathbf{Q}\right) \cap H_{r}^{1,1}$. The following lemma, which is an explicit and corrected version of the lemma on page 221 of [F1] (where there is a missing factor of $2 \pi$ in the first line of the proof), gives an upper bound for $d$, independent of $r$, completing the proof of Theorem 1 .

Lemma 1.4. Fix $0 \leq s<t<1$. Let $d$ be a positive integer and assume that all primitive $d$ th roots of unity have argument $\theta \notin(2 \pi s, 2 \pi t)$. Then $d=p_{1}^{l_{1}} \ldots p_{n}^{l_{n}}$ where the $p_{i}$ are distinct, and $p_{i}^{l_{i}} \leq 2 /(t-s)$.

Proof. We need to show that if $p^{m}>2 /(t-s)$ and $p^{m}$ divides $d$, then there exists an integer $c$ relatively prime to $d$ with $s \leq c / d \leq t$. Let $d=p^{m} \alpha$ with $p \nmid \alpha$. There exists an integer $\beta$ with $s \leq(\alpha \beta-1) / d<(\alpha \beta+\alpha-1) / d \leq t$. Both $\alpha \beta-1$ and $\alpha \beta+\alpha-1$ are relatively prime to $\alpha$ and at least one of them is relatively prime to $p$, so at least one of them is relatively prime to $d$.

\section{Example 1: Semistable elliptic surfaces}

Let $E$ be a semistable elliptic surface with $n$ singular fibers. From (1.1),

$$
\operatorname{rank} E \leq-2+\sum\left(1-e_{1, t} / 6\right)=-2+n-\sum e_{1, t} / 6=-2+n-e / 6
$$

where $e$ is the Euler characteristic of $E$ and the sums are over all the singular fibers of $E$. Since the left hand side is nonnegative, we have that $n \geq e / 6+2$. A simple computation now shows that $\gamma<1$ if and only if $n=e / 6+2$ and $E$ has singular fibers at $0, \infty$. We have the following theorem:

Theorem 2.1. Let $E \rightarrow \mathbf{P}^{1}$ be a semistable elliptic surface with Euler characteristic e. Assume that $E$ has e/6 +2 singular fibers, including singular fibers at 0 and $\infty$. Then, for all $r, \operatorname{rank} E_{r}\left(\mathbf{P}^{1}\right)=0$.

Proof. If the fiber of $E$ at $t=0$ or $\infty$ is of type $I_{n}, n \geq 0$, then the fiber of $E_{r}$ at $t=0$ or $\infty$ respectively is of type $I_{r n}$. This means that for any semistable elliptic surface, $e\left(E_{r}\right)=r e(E)$. Since the fibers at $0, \infty$ are singular, $E_{r}$ has $r e / 6+2$ singular fibers, so that $\sum f_{r, t}=r e / 6+2$. By (1.1),

$$
\operatorname{rank} E_{r}\left(\mathbf{P}^{1}\right) \leq-2+r e / 6+2-(r e / 6)=0
$$

and the result follows. 


\section{Example 2: An Elliptic modular surface}

To improve on the bounds given by Theorem 1 for the examples in this section and the next, we will need to understand explicitly how $\sigma$ acts on $H^{0}\left(\{0, \infty\}, R^{1} \pi_{*} \mathbf{C}\right)$ and on $N_{t}$, the stalk at $t$ of the pushforward of the normal bundle to a section of $\pi_{r}$, for $t=0, \infty$, so that we can compute the eigenvalues of $\sigma_{r}$ on $H_{r}^{1,1}$. In both cases, we will need to study the behavior of $\sigma_{r}$ locally at 0 and $\infty$.

Theorem 3.1. Let $E$ be defined by the Weierstrass equation:

$$
Y^{2}=4 X^{3}-27 X t-27 t .
$$

Then

$$
\operatorname{rank} E_{r}\left(\mathbf{P}^{1}\right)=\sum \phi(d)
$$

where the sum is over all $d \mid r$ such that $d \in\{1,2,3,7,8,10,12,15,18,20,42\}$.

Remark 1. The surface $E$ is an elliptic modular surface associated to $P S L_{2}(\mathbf{Z})$ (see [Sh1]), up to an isomorphism of the base. The formula was proved by Stiller [St] in the case where $r=12 n$, in general, by Shioda [Sh2], and later, in the author's Ph.D. thesis [F2]. In [Si], Silverman provides an upper bound for the rank of the group of sections of $E_{r}$ defined over a number field $K$, assuming that Tate's Conjecture is true for $E_{r} / K$.

Remark 2. We will see below that $\gamma=5 / 6$. Therefore, Theorem 1 shows that

$$
\operatorname{rank} E_{r}\left(\mathbf{P}^{1}\right) \leq \sum \phi(d)
$$

where the sum is over all $d \mid r$, with $d=2^{l_{1}} 3^{l_{2}} 5^{l_{3}} 7^{l_{4}} 11^{l_{5}}$, and $l_{1} \in\{0,1,2,3\}, l_{2} \in$ $\{0,1,2\}$ and $l_{i} \in\{0,1\}$ for $i=3,4,5$.

Proof. We first note that $E$ has a singular fiber of type $I_{1}$ at $t=1$, a singular fiber of type $I I$ at $t=0$, and a singular fiber of type $I I I^{*}$ at $t=\infty$. Thus $\gamma=5 / 6$, and $E$ satisfies the conditions of Theorem 1. Now fix $r$. Then a Weierstrass equation for $E_{r}$ is

$$
Y^{2}=4 X^{3}-27 X t^{r}-27 t^{r} .
$$

We begin by determining the local information that we will need at $t=0$. Write $r=6 k+k^{\prime}$, where $0 \leq k^{\prime} \leq 5$. Dividing through by $t^{6 k}$ we obtain the local minimal Weierstrass equation for $E_{r}$ at $t=0$ :

$$
Y_{0}^{2}=4 X_{0}^{3}-27 X_{0} t^{2 k+k^{\prime}}-27 t^{k^{\prime}},
$$

where $X_{0}=X / t^{2 k}, Y_{0}=Y / t^{3 k}$. If $k^{\prime} \neq 0$, then $E_{r}^{0}$, the fiber of $E_{r}$ at $t=$ 0 , is a singular elliptic curve, and $E_{r}$ has additive reduction at $t=0$, so that $\operatorname{dim} H^{1}\left(E_{r}^{0}, \mathbf{C}\right)=0$. For $k^{\prime}=0, E_{r}^{0}$ is a nonsingular elliptic curve with Weierstrass equation $Y_{0}^{2}=4 X_{0}^{3}-27$, and $\operatorname{dim} H^{1}\left(E_{r}^{0}, \mathbf{C}\right)=2$; so to determine the eigenvalues of $\sigma$ on $H^{1}\left(E_{r}^{0}, \mathbf{C}\right)$, we need only consider this case.

When $k^{\prime}=0,\left.\sigma\right|_{E_{r}^{0}}$ has order 6 , so for $i \neq 0 \bmod 6$ an application of the Lefschetz fixed point formula gives that

$$
\sum_{j=0}^{2}(-1)^{j} \operatorname{tr}\left(\sigma^{i}, H^{j}\left(E_{r}^{0}, \mathbf{C}\right)\right)=\#\left\{\text { points of } E_{r}^{0} \text { fixed by } \sigma^{i}\right\} .
$$


Since $\operatorname{tr}\left(\sigma^{i}, H^{j}\left(E_{r}^{0}, \mathbf{C}\right)\right)=1$ for $j=0,2$,

$$
\operatorname{tr}\left(\sigma^{i}, H^{1}\left(E_{r}^{0}, \mathbf{C}\right)\right)=2-\#\left\{\text { points fixed by } \sigma^{i}\right\} .
$$

For $i=1,5 \bmod 6, \sigma^{i}$ fixes one point of $E_{r}^{0}, 3$ points if $i=2,4 \bmod 6$ and 4 points if $i=3 \bmod 6$. Combining this information yields that the eigenvalues of $\sigma$ on $H^{1}\left(E_{r}^{0}, \mathbf{C}\right)$ are $\rho$ and $\rho^{5}$ where $\rho=\zeta_{r}^{k}$ is a 6 th root of unity.

We now compute the eigenvalues of $\sigma$ on $H_{r}^{1,1}$. By (1.2) and (1.3), we need only find $\zeta_{r}^{a_{t}}=\operatorname{tr}\left(\sigma, N_{t}\right)$, for $t=0, \infty$, and the eigenvalues of $\sigma$ on $H^{1}\left(E_{r, 0}, \mathbf{C}\right)$ and $H^{1}\left(E_{r, \infty}, \mathbf{C}\right)$ computed above. We note that (see Mi , p. 23) $N \simeq R^{1} \pi_{r *} \mathcal{O}_{E_{r}}$, so locally at $t$,

$$
N_{t} \simeq\left(R^{1} \pi_{*} \mathcal{O}_{E_{r}}\right)_{t} \simeq H^{1}\left(E_{r}^{t}, \mathcal{O}_{E_{r}^{t}}\right) \simeq H^{0}\left(E_{r}^{t}, \mathcal{O}_{E_{r}^{t}}\right)^{*} .
$$

This implies that $\operatorname{tr}\left(\sigma, N_{t}\right)$ is just the conjugate of the action of $\sigma$ on $E_{r}^{t}$.

To determine how $\sigma$ acts on $E_{r}^{t}$, we need only determine how $\sigma$ acts at a smooth point of $E_{r}^{t}$. On the fiber at $t=0$, the point $(0,1,0)$ is smooth for all $k^{\prime}$, with local affine coordinates $\left(x_{0}, z_{0}\right)=\left(X_{0} / Y_{0}, Z_{0} / Y_{0}\right)$ and local parameter $x_{0}$. We have that

$$
\sigma\left(X_{0}, Y_{0}, Z_{0}\right)=\sigma\left(X / t^{2 k}, Y / t^{3 k}, Z\right)=\left(\zeta_{r}^{-2 k} X_{0}, \zeta_{r}^{-3 k} Y_{0}, Z_{0}\right)=\left(\zeta_{r}^{k} X_{0}, Y_{0}, \zeta_{r}^{3 k} Z_{0}\right),
$$

so $\sigma$ acts on the fiber $E_{r}^{0}$ as multiplication by $\zeta_{r}^{k}$, and $a_{0}=-k=5 k+k^{\prime}$.

Now let $r=4 l+l^{\prime}, 0 \leq l^{\prime} \leq 3$. The computations at $t=1 / T=\infty$ can be carried out exactly as above. The results are summarized in Tables 3.2 and 3.3.

TABLE 3.2. Local information for $E_{r}$ at $t=0$.

\begin{tabular}{|c|c|c|c|}
\hline$k^{\prime}$ & $\begin{array}{c}\text { Local minimal } \\
\text { Weierstrass equation }\end{array}$ & $\begin{array}{c}\text { Eigenvalues of } \sigma \\
\text { on } H^{1}\left(E_{r, 0}, \mathbf{C}\right)\end{array}$ & $a_{0}$ \\
\hline 0 & $Y_{0}^{2}=4 X_{0}^{3}-27 t^{2 k}-27$ & $\rho,-\rho$ & $5 k$ \\
\hline 1 & $Y_{0}^{2}=4 X_{0}^{3}-27 t^{2 k+1}-27 t$ & - & $5 k+1$ \\
\hline 2 & $Y_{0}^{2}=4 X_{0}^{3}-27 t^{2 k+2}-27 t^{2}$ & - & $5 k+2$ \\
\hline 3 & $Y_{0}^{2}=4 X_{0}^{3}-27 t^{2 k+3}-27 t^{3}$ & - & $5 k+3$ \\
\hline 4 & $Y_{0}^{2}=4 X_{0}^{3}-27 t^{2 k+4}-27 t^{4}$ & - & $5 k+4$ \\
\hline 5 & $Y_{0}^{2}=4 X_{0}^{3}-27 t^{2 k+5}-27 t^{5}$ & - & $5 k+5$ \\
\hline
\end{tabular}

TABLE 3.3. Local information for $E_{r}$ at $t=\infty$.

\begin{tabular}{|c|c|c|c|}
\hline$l^{\prime}$ & $\begin{array}{c}\text { Local minimal } \\
\text { Weierstrass equation }\end{array}$ & $\begin{array}{c}\text { Eigenvalues of } \sigma \\
\text { on } H^{1}\left(E_{r, \infty}, \mathbf{C}\right)\end{array}$ & $a_{\infty}$ \\
\hline 0 & $Y_{\infty}^{2}=4 X_{\infty}^{3}-27 X_{\infty}-27 T^{2 l}$ & $i,-i$ & $3 l$ \\
\hline 1 & $Y_{\infty}^{2}=4 X_{\infty}^{3}-27 T^{3} X_{\infty}-27 T^{2}$ & - & $3 l$ \\
\hline 2 & $Y_{\infty}^{2}=4 X_{\infty}^{3}-27 T^{2} X_{\infty}-27 T^{4}$ & - & $3 l+1$ \\
\hline 3 & $Y_{\infty}^{2}=4 X_{\infty}^{3}-27 T X_{\infty}-27 T^{6}$ & - & $3 l+2$ \\
\hline
\end{tabular}

From Tables 3.2 and 3.3, we see that the eigenvalues of $\sigma_{r}$ on $H_{r}^{1,1}$ are exactly those $r$ th roots of unity, $e^{i \theta}$, where

$$
\theta \notin[\pi / 3, \pi / 2] \cup[3 \pi / 2,5 \pi / 3] .
$$


Thus, we have that

$$
\operatorname{rank} E_{r}\left(\mathbf{P}^{1}\right) \leq \sum \phi(d)
$$

where the sum is over all $d \mid r$ such that either $d=1$, or if $0<c<d$ and $(c, d)=1$, then $c / d \notin[1 / 6,1 / 4]$; equivalently, the sum is over all $d \mid r$ such that

$$
d \in\{1,2,3,7,8,10,12,15,18,20,42\} .
$$

To show that the above inequality is in fact an equality, note that $k=1$ implies that $H^{1}\left(\mathbf{P}^{1}, R^{1} \pi_{r *} \mathbf{C}\right)=\mathbf{C}[\mathbf{Z} / r \mathbf{Z}]$. In particular, none of the eigenvalues of $\sigma_{r}$ on $H_{r}^{1,1}$ are eigenvalues on $H_{r}^{2,0}$ or $H_{r}^{0,2}$. If $d \mid r$, an eigenspace corresponding to a primitive $d$ th root of unity is in $H^{1}\left(\mathbf{P}^{1}, R^{1} \pi_{1 *} \mathbf{Q}\right) \cap H_{r}^{1,1}$ if and only if all eigenspaces corresponding to primitive $d$ th roots of unity are also in $H^{1}\left(\mathbf{P}^{1}, R^{1} \pi_{1 *} \mathbf{Q}\right) \cap H_{r}^{1,1}$ and the above inequality is an equality. This completes the proof of Theorem 2.1.

\section{Example 3: Kummer surfaces With Picard number 20}

Let $A$ be an abelian surface, $\iota$ the involution of $A$ defined by $\iota(x)=-x$, and $E$ the resolution of the quotient surface $A /\langle 1, \iota\rangle$. Then $E$ is called the Kummer surface associated to $A$, which is an elliptic $K 3$ surface. Shioda and Inose ShI] have shown that every Kummer surface with Picard number 20 is the Kummer surface associated to the product of two isogenous elliptic curves with complex multiplication, and conversely. (They also show that 'half' of all $K 3$ surfaces with Picard number 20 are Kummer surfaces.) If the abelian surface is not the product $C_{\omega} \times C_{\omega}$, where $C_{\omega}$ is the elliptic curve with fundamental periods 1 and $\omega=e^{2 \pi i / 3}$, they show that there exists an elliptic pencil on the associated Kummer surface $E \rightarrow \mathbf{P}^{1}$ which has a section and singular fibers of type $I I^{*}, I_{1}^{*}, I_{1}^{*}$. If we assume that the fiber at 0 is of type $I_{1}^{*}$ and the fiber at $\infty$ is of type $I I^{*}$, then $\gamma=$ $(2-7 / 6)-1 / 6=2 / 3$, and Theorem 1 applies. We have the following theorem:

Theorem 4.1. Let $E_{1} \rightarrow \mathbf{P}^{1}$ be an elliptic surface with singular fibers of type $I_{1}^{*}$ at 0 and of type $I I^{*}$ at $\infty$, and exactly one other singular fiber, which is of type $I_{1}^{*}$. Then

$$
\operatorname{rank} E_{r}\left(\mathbf{P}^{1}\right) \leq \sum_{\substack{d \mid r \\ d \in\{2,3,4,5\}}} \phi(d) \leq 9 .
$$

Remark. Since $\gamma=2 / 3$, Theorem 1 shows that

$$
\operatorname{rank} E_{r}\left(\mathbf{P}^{1}\right) \leq \sum_{\substack{0 \leq l_{1} \leq 2, 0 \leq l_{2}, l_{3} \leq 1}} \phi\left(2^{l_{1}} 3^{l_{2}} 5^{l_{3}}\right) \leq 44
$$

Proof. First note that $k=2$ since away from $0, \infty$ there is one singular fiber which is of additive type. At $t=0$, a local minimal Weierstrass equation for $E_{1}^{0}$ is given by

$$
Y^{2}=X^{3}+t^{2} A_{0}(t) X+t^{3} B_{0}(t)
$$

where $A_{0}(0) \neq 0, B_{0}(0) \neq 0$ and $\operatorname{ord}_{t=0}\left(4 A_{0}(t)^{3}+27 B_{0}(t)^{2}\right)=1$. If $r$ is even, the curve $E_{r}^{0}$ is semistable of type $I_{r}$, and if $r$ is odd, $E_{r}^{0}$ is of type $I_{r}^{*}$. Let $r=2 k+k^{\prime}$ where $k^{\prime}=0$ or 1 . A computation similar to that given in the previous section shows that $a_{0}=k$ if $r$ is even, and $a_{0}=k+1$ if $r$ is odd.

If $r$ is odd, then $E_{r}^{0}$ is of additive type so that $\operatorname{dim} H^{1}\left(E_{r}^{0}, \mathbf{C}\right)=0$. If $r$ is even, then $\operatorname{dim}\left(H^{1}\left(E_{r}^{0}, \mathbf{C}\right)\right)=1$, and $\sigma_{r}$ has order 2. The eigenvalue of $\sigma_{r}$ on $H^{1}\left(E_{r}^{0}, \mathbf{C}\right)$ 
must therefore be either 1 or -1 . We will see below that 1 is an eigenvalue of both $H_{r}^{0,2}$ and $H_{r}^{2,0}$. Since $k$ is 2, it follows from (1.2) that the eigenvalue of $\sigma_{r}$ on $H^{1}\left(E_{r}^{0}, \mathbf{C}\right)$ must be -1 .

A local minimal Weierstrass equation for $E_{1}^{\infty}$ is

$$
Y^{2}=X^{3}+T^{4} A_{\infty}(T) X+T^{5} B_{\infty}(T)
$$

where $B_{\infty}(0) \neq 0$. Letting $r=6 l+l^{\prime}$ with $0 \leq l^{\prime} \leq 5$, we find that $a_{\infty}=5 l+l^{\prime}-1$ if $l^{\prime} \neq 0$ and $a_{\infty}=5 l$ if $l^{\prime}=0$. Also, if $l^{\prime}=0$, the eigenvalues of $\sigma$ on $H^{1}\left(E_{r}^{\infty}, \mathbf{C}\right)$ are $\rho$ and $\rho^{5}$. If $l^{\prime} \neq 0$, then $\operatorname{dim}\left(H^{1}\left(E_{r}^{\infty}, \mathbf{C}\right)\right)=0$.

Combining the above information with (1.2) and (1.3), we see that the eigenvalues of $\sigma$ on $H_{r}^{1,1}$ are $\zeta_{r}^{-a_{\infty}}, \ldots, \zeta_{r}^{a_{\infty}}$ if $6 \mid r$, and $\zeta_{r}^{-a_{\infty}+1}, \ldots, \zeta_{r}^{a_{\infty}-1}$ if $6 \mid r$. In particular, the eigenvalues of $\sigma$ on $H_{r}^{1,1}$ are the $r$ th roots of unity, $e^{i \theta}$, with $\theta \notin[5 \pi / 3, \pi / 3]$, and Theorem 4.1 follows.

\section{ACKNOWLEDGEMENTS}

A slightly weaker version of Theorem 1 is contained in the author's Ph.D. thesis, as is Example 2. I am very grateful to Serge Lang, Joe Silverman and Ian Grojnowski for their help, encouragement and guidance.

\section{REFERENCES}

[CZ] Cox, D., Zucker, S., Intersection Numbers of Sections of Elliptic Surfaces, Inv. Math. 53 (1979), 1-44. MR 81i:14023

[F1] Fastenberg, L., Mordell-Weil groups in procyclic extensions of a function field, Duke Math. J. 89 (1997), 217-224. MR 98f:11058

[F2] Fastenberg, L., Mordell-Weil groups in procyclic extensions of a function field, Ph.D. Thesis, Yale University, 1996.

[Mi] Miranda, R., The Basic Theory of Elliptic Surfaces, Dottorato Ric. Mate., ETS Editrice, Pisa, 1989. MR 92e:14032

[Sh1] Shioda, T., On Elliptic Modular Surfaces, J. Math. Soc. Japan 24 (1972), 20-59. MR 55:2927

[Sh2] Shioda, T., An Explicit Algorithm for Computing the Picard Number of Certain Algebraic Surfaces, Amer. J. Math. 108 (1986), 415-432. MR 87g:14033

[ShI] Shioda, T., Inose, H., On Singular K3 Surfaces, Complex Analysis and Algebraic Geometry (1977), 119-136. MR 56:371

[Si] Silverman, J., A Bound for the Mordell-Weil Rank of an Elliptic Surface after a Cyclic Base Extension, J. Algebraic Geom. 9 (2000), 301-308. MR 2001a:11107

[St] Stiller, P., The Picard Number of Elliptic Surfaces with Many Symmetries, Pacific J. Math. 128 (1987), 157-189. MR 88c:14054

Department of Mathematics, Yeshiva University, New York, New York 10033

E-mail address: fastenb@ymail.yu.edu 